\title{
Update on liquid biopsy in clinical management of non-small cell lung cancer
}

This article was published in the following Dove Press journal:

OncoTargets and Therapy

\author{
Zhen Wu \\ Zhen Yang \\ Yu Dai \\ Qiang Zhu \\ Liang-An Chen \\ Respiratory Department, Chinese PLA \\ General Hospital, Beijing, People's \\ Republic of China
}

\begin{abstract}
Lung cancer, a leading cause of cancer-related mortality, has a low rate of early diagnosis and a poor prognosis for advanced stages. Recent advances in further mastery of the biology of tumors promote the diagnosis and therapy, especially for non-small cell lung cancer (NSCLC). However, tumor tissue-based information is often not available in most cases due to the invasive and high risk nature of the tumor biopsy procedures. Liquid biopsy, based on the multiple liquid samples including circulating tumor cells (CTC), circulating tumor DNA (ctDNA), and tumor-derived exosome obtained from blood or urine as well as other body fluids, can also provide valuable tumor-related information, playing an important role in management of NSCLC in clinical practice. It is widely believed that concordance of detection for tumor by liquid samples in comparison with tissue biopsy for both early and advanced stage NSCLC patients is optimistic. We herein review the current and future clinical application of liquid biopsy, including early diagnosis and management of precise personalized treatment in lung cancer. The future directions of development for liquid biopsy are also discussed in this review.
\end{abstract}

Keywords: liquid biopsy, non-small cell lung cancer, early diagnosis, molecular targeted therapy, immunotherapy

\section{Introduction}

Lung cancer is the most common cause of cancer-related mortality in the world, with 154,050 related new deaths in America every year reported in 2018. ${ }^{1,2}$ Extremely low rates of early diagnosis lead to most patients being diagnosed with advanced stage, and the 5-year relative survival rate remaining at only $18 \%$, which contribute to the high mortality of lung cancer. ${ }^{1}$ Therefore, it is important to promote pre-screening among the general population to detect lung cancer in earlier stages. A low-dose CT scan has been recommended in screening for lung cancer since 2011 as it can reduce the mortality rate of $20 \%$ from lung cancer and the mortality rate of $6.7 \%$ from any cause. ${ }^{3}$ However, its clinical practice value is limited by the false positive caused by image detection, leading to unnecessary invasive operations in the healthy population. ${ }^{3}$ Also, long-term annual CT scanning inevitably increases the risk of radiation exposure. There is an urgent need for more specific and less invasive biomarkers that can be used as complementary or alternatives to radiological approaches to better select the right risk cohort. Liquid biopsy, based on body fluids including plasma, urine, and other liquids, can detect tumor-related biomarkers to diagnose lung cancer earlier and safer. ${ }^{4}$ Moreover, the combination of liquid biopsy and radiology examination would be able to increase the accuracy and costeffectiveness of screening and early detection for lung cancer.
Respiratory Department, Chinese PLA General Hospital, 28 Fuxing road, Haidian district, Beijing 100853, People's Republic of China

Tel +860 1055499327

Email chenliangan30I@I63.com 
The individual precise targeted treatment based on molecular classification has increasingly improved the overall survival of lung cancer, especially the NSCLC, significantly. ${ }^{5,6}$ For example, EGFR tyrosine kinase inhibitors have increased the overall survival of patients with EGFR mutated significantly. ${ }^{5}$ Except the molecular targeted therapy, immune therapy is also increasingly important among advanced lung cancer and promotes the outcomes of advanced NSCLC. The selection criterion for immune therapy is recommended to be based on the molecular status of PD-L1 expression. ${ }^{7}$ Other potential factors, including tumor mutation burden (TMB), are also analyzed to predict the prognosis of patients receiving immune therapy according to the genomic profiles of the tumor. ${ }^{8,9}$ Tissue biopsy was regarded as the "standard procedure" for molecular detection and was indispensable in decision-making concerning treatment for advanced NSCLC patients before 2016. ${ }^{10}$ However, tumor tissue is often not available due to the invasiveness and the failure of getting enough tumor tissue for further detection of gene variations. ${ }^{11}$ Especially for those targetedtherapy resistant patients, re-biopsy is extremely difficult due to the suboptimal clinical condition. ${ }^{12}$ Liquid biopsy, characterized by non-invasiveness, easy accessibility, and good repeatability, was increasingly used to conduct molecular profiling testing and monitoring drug-resistance dynamically. ${ }^{13,14}$ A large variety of surrogates in human liquid are available to make the individual therapy more efficientand precise.

In this paper, we will focus on clinical applications and future directions of liquid biopsy in management of NSCLC.

\section{Early diagnosis of lung cancer by liquid biopsy}

It has been determined that liquid biopsy is likely to detect tumor-specific biomarkers to diagnose lung cancer at early stage in previous research. Actually, different tumor-derived elements, including ctDNA, cfRNA, CTC, exosomes, and protein can be isolated from body fluids to reveal the molecular landscape of the tumor. In previous research, it was found that the level of plasma cfDNA in cancer patients is higher in comparison with healthy controls. ${ }^{15}$ In addition, it was not only confirmed that the concentration of cfDNA in plasma was higher in NSCLC patients compared to benign lung tumors and healthy controls, but also demonstrated that cfDNA integrity obtained from $\mathrm{Ct}$ values of 100-bp qPCR products divided by ones in 400-bp, showed great power ( $91 \%$ sensitivity, $68.2 \%$ specificity) to discriminate NSCLC and benign lung tumors. ${ }^{16}$ It was shown that mutations in ctDNA only has $50 \%$ sensitivity of early detection for lung cancer due to the fewer detectable amounts of ctDNA released by early-stage tumors, while proteins in plasma have also been described to be useful to detect and diagnose lung cancer at early stages. Therefore, an evaluation of a combination of 16 driver genes mutation in ctDNA and eight circulating proteins, including carcinoembryonic antigen (CEA), cancer antigen 125 (CA-125), cancer antigen 19-9 (CA19-9), hepatocyte growth factor (HGF), tissue inhibitor of metalloproteinases 1 (TIMP-1) protein levels, pro-lactin (PRL), osteopontin (OPN), and myeloperoxidase (MPO), called CancerSEEK, was conducted to improve the early detection for multiple cancers, including lung cancer. The results presented that CancerSEEK had the ability not only to diagnose the presence of relatively early cancers with higher sensitivity, $70 \%$ for median and $60 \%$ for lung cancer, but also to differentiate the original organ of cancers effectively. ${ }^{17}$

Non-coding RNAs in biologic fluids have been investigated to assess its potential to detect lung cancer at the early stage. MiRNAs are the most adequate cfRNA molecules in the fluids samples, and various studies have proposed this as a useful and non-invasive tool for cancer diagnosis. Different expression of a panel of miRNAs in sputum, including miR-145, miR-126, and miR-7, generated $90 \%$ sensitivity and specificity to distinguish NSCLC from the controls. ${ }^{18}$ In addition to identifying malignant or benign, miRNAs in plasma are also used to distinguish the histological classification of lung cancer, whether it is small cell lung cancer or non-small cell lung cancer. Plasma panel A, consisting of six miRNAs (miR-17, miR-190b, miR-19a, miR-19b, miR-26b, and miR-375), demonstrated high power to discriminate the lung cancer from healthy donors, while plasma panel $\mathrm{B}$, involving three miRNAs (miR-17, miR-190b, and miR-375) from panel A determined high diagnostic accuracy in distinguishing between small cell lung cancer (SCLC) and NSCLC. ${ }^{19}$ This means that non-invasive biomarkers could play a crucial role in diagnosing as well as determining optimal treatment based on benign and malignant differentiation and histological judgment.

Epigenetic biomarkers, including cfDNA/RNA methylation, have gained increasing attention as a non-invasive material for lung cancer early diagnosis. Abnormal DNA 
methylation was often found to be associated with cancer, while hypermethylation was usually discovered in tumorsuppressor genes and hypomethylation in protooncogenes. $^{20}$ It has been reported that DNA methylation can happen at an early stage of lung cancer and be used for the screening and early diagnosis of lung cancer. Methylation of SHOX2 and PTGER4 were demonstrated to be able to distinguish lung cancer from healthy control, with $91-98 \%$ area under curve (AUC). ${ }^{21}$ Based on the specific enrichment of methylated fragments from cfDNA and its tumor-specific patterns, a high AUC of 0.971 was produced to discriminate between early stage lung cancer and healthy controls. ${ }^{22}$ CfRNA methylation was demonstrated to mediate miRNA expression and cancer cell migration. ${ }^{23}$ Serum miR-34b/c methylation was applied to diagnose malignant pleural mesothelioma, ${ }^{24}$ and cfRNA methylation for early diagnosis of lung cancer needs further study to prove.

Metabolites, including amino acids, carboxylic acids, and tocopherols are often downregulated in cancer samples, which can be adopted to diagnosis cancer at an early stage. A classifier of nine serum metabolites allowed us to distinguish cancer with control samples with a sensitivity of $100 \%$ and specificity of $95 \%{ }^{25}$ It has been proved that phosphatidylethanolamines (PE) can be used to distinguish benign and malignant nodules within a CT screening trial. ${ }^{26}$

Imaging examinations, like CT scans, were identified as a useful way to screen and detect lung cancer at an early stage. However, the application was limited by the higher false positive rate and radiation exposure. Given noninvasive biomarkers' wide applications in screening of lung cancer, a combination of those non-invasive biomarkers and radiologic screening, like a CT scan, is more valuable and acceptable than using the CT scan alone. A panel of three miRNAs selected from the TCGA database demonstrated a sensitivity of $81.2 \%$ to discriminate lung cancer from healthy controls, while two miRNAs from the panel combining the measurement of size of pulmonary nodules can reach $89.9 \%$ diagnostic sensitivity. ${ }^{27}$ In addition to panels of miRNAs, autoantibody assays have also been determined to be capable of complementing CT scanning in lung cancer diagnosis as they can be found in plasma 5 years before the initial detection of lung cancer. ${ }^{28}$ A panel of seven autoantibodies (7-AABs) was determined to have $61 \%$ sensitivity and $90 \%$ specificity in patients with solid and/or nodules ground glasses GGNs), which were higher than traditional biomarkers such as CEA. The positive predictive values (PPVs) in the 7-AABs panel and CT scanning were $85.2 \%$ and $69.0 \%$, respectively. The combination of CT scanning and 7-AABs panel significantly elevated the PPV in comparison to a CT scan alone $(95.0 \%$ vs $69.0 \%$; $P<0.001)$ or 7 -AABs panel alone $(95.0 \%$ vs $85.2 \% ; P<0.001)$. Moreover, the false positive rates in patients with defined size and GGNs and/or nodules decreased significantly by combining CT scanning and the 7-AABs panel together. ${ }^{29}$

To summarize, those non-invasive biomarkers analyzed by liquid biopsy provide an efficient way to screen and find lung cancer at an early stage. Moreover, when those biomarkers are combined with imaging examination, there would be more lung cancers detected at early stages than before. Therefore, all of these deserve a wide range of applications in clinical practice.

\section{Utility of liquid biopsy in management of molecule targeted therapy}

One of the latest breakthroughs in NSCLC is represented by the molecule-targeted therapy in specific molecular status of this disease. Also, the most important targeted therapies are EFGR tyrosine kinase inhibitors (EGFR-TKIs) and ALK receptor tyrosine kinase (ALK-TKIs), which have improved the survival of advanced NSCLC significantly in comparison with chemotherapy. ${ }^{5,6,30,31}$ However, drug-resistance is inevitable during the course of management of these targeted therapies. ${ }^{32}$ The next-generation drugs able to overcome those resistance mechanisms have also been developed rapidly. According to the NCCN guidelines, testing gene variations is required before both choosing proper treatment for newly-diagnosed and drug resistant NSCLC patients. ${ }^{10}$ However, many patients have no access to receive tumor biopsy due to the invasiveness of the procedure or the tumor tissue obtained is not sufficient for detection of gene alterations. ${ }^{33}$ Especially for drug resistant patients, $20 \%$ of these have no access to successful rebiopsy. ${ }^{34}$ Moreover, tumor heterogeneity makes the tumor biopsy in one site not able to cover the comprehensive genomic profiles and which can be complemented by plasma genotyping as it is circulating throughout the body and evenly distributed. ${ }^{35}$ Finally, it is difficult to achieve dynamic monitoring during the treatment process due to the invasive nature of tissue testing. Previous researchers have determined that liquid samples, including plasma and urine, can be used as a surrogate for tissue to test gene variations, 
and can dynamically monitor changes to analyze drug resistance earlier during the course of treatment, as part of the circulating nucleic acid in it is tumor-related and easy to be obtained and operated. ${ }^{36-38}$ As previously mentioned, EGFR and ALK are the major targeted molecules for precise targeted therapy, and the majority of liquid biopsies involved research concerns these two molecules.

\section{Effective detection of genotype to select proper patients for targeted therapy}

Gene mutation based on tumor tissue-testing can predict tumor response to targeted treatment, and is often viewed as gold standard, while liquid biopsy can also provide an efficient way to analyze gene mutation for proper therapy when tissue biopsy is not available. Recent progress in the sensitivity and accuracy of DNA detection has enabled the genotyping of ctDNA, mostly consistent with genomic variations found in tumors. Since ctDNA has been discovered in the plasma of NSCLC patients, there were increasing numbers of researchers detecting gene variations via plasma: it was found for the first time that $79.7 \%$ of EGFR mutation in plasma can be detected in matched tumor DNA by the method of denaturing highperformance liquid chromatography (DHPLC) in advanced NSCLC patients. ${ }^{39}$ Using a more sensitive amplification refractory mutation system (ARMS) to quantitate ctDNA in advanced NSCLC patients, it has been proved that plasma could be a suitable surrogate for EGFR mutation detection, since high concordance, specificity, and sensitivity have been achieved. ${ }^{40}$ It was also recommended by the official organizations to detect the targeted molecular by cobas ${ }^{@}$ (a common method of ARMS) when tissue is not accessible. ${ }^{10}$ Concordance between ctDNA and tumor DNA in terms of genetic detection is increasing with the advance in testing methods. In comparison with tumor biopsy, sensitivity of detection for EGFR exon19 deletions and exon21 L858R were $81.82 \%$ and $80.00 \%$ through droplet digital PCR assays, respectively. ${ }^{41}$ Liquid biopsy can not only detect the gene mutations non-invasively, but also evaluate the prognosis for targeted therapy. Studies have demonstrated the EGFR mutation in plasma could predict outcomes independently for patients receiving the therapy of gefitinib, since the results of ORR and PFS, which were similar to previous results presented by tumor tissue. ${ }^{42}$

In contrast to EGFR, the applications of liquid biopsy in ALK mutations are limited. Almost all these tests were based on the NGS method because it was difficult to measure the multiple rearrangements through ctDNA with PCR-based methods. NGS reached an acceptable level of sensitivity of $79.2 \%$ and specificity of $100 \%$ for detection of ALK mutations in plasma ctDNA of newlydiagnosed patients with confirmed ALK rearrangements in their tissue biopsies. ${ }^{43}$ Besides the optimal sensitivity, capture-based NGS can also detect rare novel ALK rearrangements like FAM179A-ALK and COL25A1-ALK in plasma ctDNA. ${ }^{44}$ There are also other driver genes like MET, ROS1, and BRAF in NSCLC, but fewer studies existed to detect these genetic aberrations in peripheral blood samples. With the presence of various methods for liquid biopsy and the elevation in diagnostic accuracy of ctDNA, reliable technology allows for detection of multiple genes in parallel for an increasing number of gene targets identified as a therapeutic focus. Multiple genes variations can be analyzed with optimal sensitivity in plasma at the same time by using next generation sequencing (NGS). It has been found that overall sensitivity of alterations in plasma cfDNA can achieve $94 \%$ by using a NGS panel of 35 cancer-related genes. ${ }^{45}$

In addition to being consistent with tumor biopsy, some of the results discovered by ctDNA but not in tumor tissue were also likely to be demonstrated as meaningful in some cases. For example, drug resistant mutation T790M can be found in plasma but not in tumor, and the outcome of following therapy reflected the accuracy of this result provided by plasma ctDNA only. ${ }^{46}$ It was believed that existing tumor heterogeneity generated such a discrepancy between ctDNA and tumor tissue DNA, which meant the individual biopsy of a single site could not cover the whole genomic profile of the tumor itself. Besides the analysis of targeted molecular, gene alterations detected by plasma ctDNA can also predict the outcome of therapy.

\section{Dynamic follow-up to predict the efficacy and outcome of targeted therapy}

Clinical use of dynamic evaluation of driver gene alterations qualitatively or quantitatively was able to predict the survival for targeted therapy. It is generally believed that the status or frequency of EGFR mutations in plasma is associated with therapeutic efficacy in people with positive sensitizing EGFR and receiving 1st EGFR-TKI. There were reports on the relationship between EGFR mutation status at baseline and the overall prognosis of EGFR-TKI therapy. It was proved by $\mathrm{Zhu}$ et $\mathrm{al}^{47}$ that lower plasma EGFR mutation concentrations in baseline were correlated 
with longer progression-free survival (16.23 months vs 8.43 months; $P=0.0019)$. However, there also existed the opposite results. Scientists found that EGFR mutation $(+)$ can predict a better progress free survival (PFS) than EGFR(-) in plasma (18.8 vs 9.4 months, $P=0.003){ }^{48}$ Patients with EGFR $(+)$ in ctDNA superior PFS (12.6 vs 6.7 months, $P<0.001)$ and OS (35.6 vs 23.8 months, $P=0.028)$ were compared with those with $\operatorname{EGFR}(-)$ in ctDNA. Moreover, it was also shown that patients harboring high EGFR-mutated abundance $(>5.15 \%)$ in ctDNA presented longer PFS than those having low EGFR mutated abundance $(\leq 5.15 \%)$ in plasma (15.4 vs 11.1 months, $P=0.021){ }^{49}$ There are corresponding reasons accounting for these different results. For the former ones, the presence of EGFR mutation of higher allelic frequency suggested a heavy tumor burden compared to the negative and lower allelic frequency for EGFR alterations in plasma. The extensive tumor burden may lead to progress at a faster rate, which lead to a shorter survival for 1st EGFR-TKI in patients with EGFR $(+)$ in plasma. ${ }^{47}$ For the latter, the relatively high allelic frequency of intratumor EGFR mutant could suggest that TKIs-sensitive mutated clones accounted for the majority of whole tumor clones, and took a longer time to develop drug resistance compared to those harboring low frequency of intra-tumor EGFR mutations. ${ }^{48,49}$ Nevertheless, the mechanism of ctDNA released into the circulatory system is not clear. The detection assays used in these studies to analyze the EGFR mutation qualitative and quantitatively were also different, which maybe influence the comparison among these results concerning the prognosis value of EGFR mutation. Therefore, the relationship between EGFR mutation in ctDNA and outcomes of EGFR-TKI still needs further exploration.

Dynamic following-up of the mutated genes such as EGFR during the course of targeted therapy appears to be associated with outcome of involved treatment. It has been observed that PFS was 10.1 months for patients with undetectable EGFR mutation in plasma after receiving EGFR-TKI for 2 months, while it was only 6.3 months for those with detectable EGFR mutation $(\mathrm{HR}=3.88,95 \% \mathrm{CI}=1.48-10.19$, $P=0.006) .{ }^{50}$ A prospective study by Zhou et $\mathrm{al}^{51}$ found two dynamic types of quantitative changes of L858R during the course of EGFR-TKI treatment, one that rose to its highest level (ascend type) while the other maintained its stable level (stable type) when disease progressed. They found that median PFS in the ascend type was higher than in the stable type (11.1 vs 7.5 months, $P=0.023$ ). The methylated ctDNA has also been investigated to be related with the prognosis of EGFR-TKI. Salazar et $\mathrm{al}^{52}$ proved that patients with methylated CHFR promoter survived for a shorter period when receiving EGFRTKI as the second-line therapy, compared to conventional chemotherapy. Wang et $\mathrm{al}^{43}$ also discovered that the rate of ALK rearrangements drops to $69.2 \%$ after treatment with Crizotinib. Decreased allele frequency of BRAF V600E by dynamic monitoring in plasma ctDNA was correlated closely to corresponding targeted treatment response. ${ }^{53}$

In conclusion, dynamic monitoring of quantitative and qualitative changes in variations is mainly for prediction of outcomes for targeted treatment in patients with positive driver gene alterations. It can also provide the chance of finding a drug resistant mechanism in advance during the process of surveillance.

\section{Early finding and exploring mechanism of drug resistance in targeted therapy}

There is no doubt that drug resistance usually happens after long-term use of targeted therapy. Many common mechanisms of resistance have been identified, including T790M for first and second line of EGFR-TKI and C797S for third line of EGFR-TKI. The selection for appropriate therapy after drug resistance is based on the best known of these mechanisms. However, many advanced patients have no access to re-biopsy for analysis of drug resistant mechanism due to the poor health condition. The merge of liquid biopsy enables these resistant mechanisms to be analyzed easily and earlier. Previous research demonstrated that $45 \%$ of patients with $\mathrm{T} 790 \mathrm{M}$ could have this variation detected before progression of the disease. ${ }^{54}$ It has been determined that T790M can be found in plasma ctDNA prior to radiological progression as much as 2-12 months earlier. ${ }^{50}$ T790M can also be detected in urine ctDNA 3 months before disease progression confirmed by radiologic examination. ${ }^{55}$ In addition to early detection, the concordance between cfDNA and tumor tissue in the analysis of T790M is also considerable to guide the next generation line of therapy. T790M was detected in cfDNA with sensitivity from 70-83\% when compared with tumor re-biopsy. ${ }^{56-58}$

Moreover, patients with $\mathrm{T} 790 \mathrm{M}(+)$ in plasma could predict an optimal survival for osimertinib. Research has also shown that patients with $T 790 \mathrm{M}$ positive in plasma have equivalent outcomes with patients positive by tumor biopsy after receiving osimertinib. ${ }^{56}$ However, in the group of patients with $\mathrm{T} 790 \mathrm{M}(+)$ in tumor tissue and receiving TKI treatment at the 2nd line or later, further analysis showed that $\mathrm{T} 790 \mathrm{M}$ positivity in plasma would be a prognostic factor for the worse OS during 
receiving the 2 nd line or later TKI treatment after 1 st $\mathrm{PD}$, and the group with T790M positive in ctDNA had significantly shorter overall survival (OS) than the negative group (26.9 months vs NA, $P=0.0489) .{ }^{54}$ Dynamic monitoring of T790M can also be beneficial to guide an efficient management of treatment. The complete clearance of T790M during the treatment with osimertinib would predict a worse PFS (6.1 months vs 15.2 months). ${ }^{59}$ Thress et al. ${ }^{60}$ also found that the continual appearance of T790M after 6 weeks' treatment with osimertinib would predict a shorter PFS (5.5 months vs 10.9 months) and decreased ORR (35\% vs 70\%). These results suggest the emergence of pre-existing distinct resistant clones after drug resistance of osimertinib, which lead to a worse prognosis.

Similarly, resistant alterations in patients proceeding with osimertinib are continually emerging. MET amplification and $\mathrm{C} 797 \mathrm{~S}$, the most common mechanism for drug resistance for third generation EGFR-TKI - osimertinib, were also discovered through analyzing ctDNA in plasma. ${ }^{32,61}$ In addition to these, a growing number of mechanisms were found in ctDNA from multiple biologic liquid samples. Point mutations like EGFR G796/C797, L792, and L718/G719 were detected in the plasma of drug-resistant patients. ${ }^{62,63}$ BRAF V600E has been demonstrated as a resistant mechanism via pleural effusion in patients found to be drug resistant with osimertinib. ${ }^{64}$

There was also evidence to demonstrate that resistant mutations of crizotinib appeared 2 months before radiographic disease progression. ${ }^{65}$ The drug resistant mutation for ALKTKI, including L1196M and G1269A, can also be analyzed by ctDNA in biologic fluids. Also, dynamic changes in these resistant mutations are also consistent with the efficacy of the 2nd line of ALK-TKI. ${ }^{66}$ Other mechanisms, like I1171T and V1180L mutations, were also detected in ctDNA by NGS. ${ }^{67}$ Although acquired resistance mutations for ALK-TKI are not required to change primary therapy into another different ALK-TKI forwards, it is important to determine the next generation of TKIs based on the distinct mutations. When the re-biopsy is not available, the genomic profile obtained from ctDNA by NGS panel is preferred as it no only can provide the known mechanisms but also provide the other unknown and potential mechanisms of resistance for which proper treatment may be acquired from clinical trial or expanded access.

\section{Evaluation for immunotherapy via liquid biopsy}

In recent years, immune checkpoint inhibitor (ICI) therapy has achieved great success in multiple malignant solid tumors, especially for NSCLC. It not only revolutionizes the treatment strategy for NSCLC, but also prolongs the survival for advanced patients. However, the ORR of ICI therapy only remains at $20 \%$ or less in second line treatment. Identification of immune-related biomarkers applied for diagnosis, prognosis, and monitoring during immunotherapies is urgently required. How to choose patients appropriately for ICI therapy has become a hot issue nowadays. Researchers have shown that the high expression of PD-L1 protein presented in tumor cells was determined to be related with efficacy of ICI treatment. ${ }^{68}$ Easy access to body fluids makes it preferred to tumor biopsy concerning the detection of biomarkers as it has the ability to cover molecular landscape of tumors non-invasively and dynamically. And the CTCs could represent a surrogate for analysis of PD-L1 expression. It was found that expression of PD-L1 on the surface of CTCs both at baseline and 3 months after treatment were found to be associated with poor patients' outcome. ${ }^{69}$ Also, PD-L1 expression in CTCs and peripheral WBCs were correlated with PD-L1 expression in tumor tissue, with concordances of $93 \%$ and $80 \%$, respectively. ${ }^{70}$ Ilie et al $^{70}$ also confirmed that analysis of PD-L1 on CTCs is highly feasible, but the expression of PD-L1 on CTCs has no significant impact on the prognosis for immunotherapy. Therefore, there remain conflicting results in different cohorts for the lack of standardized methodology for detection on PD-L1 expression. ${ }^{71}$ Further exploration needs to illustrate the relationship between PD-L1 expression on CTCs and prognosis for immunotherapy.

In addition to PD-L1 expression on tumor cells, tumor mutation burden (TMB), measured by mutations per megabase (mb) with hybrid based NGS, might be another response biomarker for outcomes of immunotherapy. An analysis from the trial of CheckMate-026 determined a relationship between high tissue-based TMB and better clinical benefit with nivolumab in NSCLC in the firstline. ${ }^{9}$ Aaron et $\mathrm{al}^{8}$ also provided evidence to show that high TMB ( $\geq 20$ mutations $/ \mathrm{mb}$ ) predicted better outcomes, including ORR (46\% vs $14 \% ; P=0.0025)$, PFS (10 vs 2.2 months; $P=0.0005)$, and OS(11.1 months vs not reached, $P=0.0557)$ in comparison with low ( $1-5$ mutations $/ \mathrm{mb})$ to intermediate (6-19 mutations/mb) TMB after receiving treatment with anti-PD1/PD-L1 monotherapy. However, obtaining the tumor tissue needed for mutations detection is always a challenge facing the advanced NSCLC patients. Some researchers have determined that blood also can be considered as a source of molecular testing. The number of mutations in variants of unknown 
significance (VUS) in plasma ctDNA was shown to be related with outcomes of checkpoint inhibitor-based immunotherapy. Khagi et $\mathrm{al}^{72}$ presented that PFS and disease control rate (DCR) were improved in patients with VUS $>3$ compared to those with VUS $\leq 3$. Also, responders had a higher median PFS than non-responders with VUS $>3$ ( 23 vs 2.3 months, $P=0.0004$ ). With the deep exploration in alteration detection by cfDNA, calculation of TMB from blood (bTMB) cfDNA has been developed through deep sequencing. Research has shown that bTMB is correlated to clinical prognosis for immune checkpoint inhibitor therapy. Analysis found bTMB analyzed by the NGS panel with a robust cutpoint $\geq 16$ performed in POPLAR samples and confirmed in OAK samples predicted an increased PFS benefit from atezolizumab. Also, bTMB was determined to be associated with tissue TMB (tTMB) not the PD-L1 expression. ${ }^{73}$ All of these suggest that the value of this bTMB assay to conduct molecular diagnostic and therapeutic algorithms for immunotherapy is promising, and the prospective validation of bTMB assay in the first line of NSCLC is warranted.

Dynamic monitoring of identical molecules was also crucial during the process of immunotherapy. The changes of ctDNA levels predict the efficacy of immunotherapy. It was presented that PFS was longer in ctDNA responders ( $>50 \%$ decrease at mutant allele fraction in comparison with baseline) than non-responders (205.5 vs 69 days; $P<0.001) .{ }^{74}$ It has also been proved that the patients with ctDNA detected at 8 weeks after ICI means poor PFS and OS outcomes than patients with undetected ctDNA at the same time. ${ }^{75}$ An imaging scan is insufficient to evaluate the efficacy and analyze the drug resistance, since the pseudoprogression-appearance of larger or new tumor lesions often happen before the tumor actually shrinks. Evidence has been provided to show that dynamic monitoring of ctDNA can be applied to distinguish real progression of disease from pseudo-progression, since the ctDNA levels decrease rapidly in pseudo-progression, while patients with real progression shows significant increases in ctDNA levels. ${ }^{76}$ Monitoring the efficacy and disease through liquid biopsy enables the ICI more efficiently and successfully.

More broadly, an integrated and comprehensive biosampling has been approached to find non-invasive biomarkers for administration of immunotherapy. However, the way to detect expression of PD-L1 on CTCs still remains the big issue, and the method to measure the bTMB is distinct from tTMB and has not come to a conclusion yet. Standardization of methods and rigorous validation are required to better understand the relationship between biomarkers and immunotherapy.

\section{Future directions}

Liquid biopsy is an increasingly developing field in oncology, especially for lung cancer. However, there still remain some challenges for the sensitivity and utility of liquid biopsy in clinical practice, as the percent of circulating tumor related molecules is scarce. The accurate mechanism of the releasing of ctDNA has not been revealed exactly, and the percentage of ctDNA in circulating free DNA (cfDNA) is often as low as $1 \%$ in most cases. Therefore, the technology needed to evaluate and analyze the molecular profiles in liquid biopsy must be sensitive and standardized. With the development of accurate detection methods such as Next-Generation Sequencing (NGS); Magnetics (BEAMing); and digital driplet PCR (ddPCR), liquid biopsy has made it possible to detect the driver gene mutations at extreme rarity and cover the cancerrelated driver genes as much as tumor tissue can. ${ }^{36,77,78}$

Although plasma is the most popular fluid sample for liquid biopsy, it is not always preferred in all cases. There are also many other specimens available, like urine, sputum, cerebrospinal fluid (CSF), or pleural effusion, to address liquid biopsy under different situations. ${ }^{79,80}$ Easy access to liquid samples has also ensured the dynamic monitoring of the efficacy and drug resistance during the process of treatment. ${ }^{50,81}$ Each of these modalities is able to present novel diagnostic information, and their role of exploration is highly valued.

Tumor heterogeneity is another issue needed to be considered during the whole management of lung cancer. A single type of sample, whether tumor tissue or liquid fluid, might not be able to cover all relevant distinct subclones. Various mediums, including ctDNA, CTC, and exosomes, allow for tumor analysis from different aspects. A variety of biologic liquid samples are different and complementary to illustrate the molecular profiles comprehensively. Here, we summarize the most important aspects of liquid biopsy which would be progressed in the future.

\section{Advanced methods developed for liquid biopsy}

Various analytic methods have been developed to identify molecular alterations through liquid biopsy. Sensitivity is the most important factor to be considered, as the percentages of tumor related ctDNA vary from patient to patient and are often extremely limited in most cases. ddPCR, an absolute 
quantification assay of molecular identification, makes the concordance of detection of mutation skyrocket in comparison with tumor biopsy. ${ }^{41,82}$ It was determined that ddPCR was more sensitive than conventional PCR based assays through a technology to part the sample to a single droplet with 1 or 0 DNA molecules to be amplified. The results were confirmed by analyzing the signal from each positive or negative droplet. $^{83}$

In addition, the covered range of molecules to be detected is another key factor to be measured. As we all know, classic PCR-based assays are only able to target the known driver mutations. It is recommended that cobas ${ }^{\circledR}$ test the exon 19 deletions, L858R and T790M mutations from ctDNA. Recent studies have frequently applied more accurate analyses both in depth and breadth to cover tumor-related driver gene mutations as much as possible, from a panel of targeted regions of exons or introns in various genes, whole exomes sequencing, to whole genomes sequencing. Especially for drug resistant mechanism exploring, only NGS can make it possible to detect the potential mechanisms. A prospective study performed by Thompson et $\mathrm{al}^{36}$ showed that over 50 therapeutically targetable driver mutations and 12 resistance mutations can be detected simultaneously in ctDNA by NGS, and the unique mutation identified can also guide an effective therapy when tissue biopsy is not feasible. With the emphasis on co-mutant variations, along with therapy-targeted molecules including EGFR and ALK, NGS enables comprehensive evaluation of precise treatment with the detection of multiple genes alterations at the same time. ${ }^{84}$ Especially with additional barcoding technology, the sensitivity would be increasing to lower the limit of mutation frequency for detection. ${ }^{85,86}$ Many researchers and statements recommend the NGS supporting the use of NGS in clinical practice. ${ }^{87,88}$ Except for the developments in methods aimed at assay detection, more efforts have been made to improve the efficacy of ctDNA accumulation in liquid samples. Mouliere et $\mathrm{al}^{89}$ revealed the size difference between non-mutant and mutant DNA by a genome-wide and pancancer scale. Enrichment of ctDNA exists in fragment sizes between 90 and 150 bp. Choosing fragments between 90 and $150 \mathrm{bp}$ increases the detection of alterations, including the actionable mutations and copy number variations, with a area under the curve (AUC) $>0.99$ in comparison with AUC $<0.80$ without fragmentation selection.

Various mediums available for liquid biopsy At present, the most common and mature biologic element for liquid biopsy is ctDNA, which is released into blood circulation through apoptosis or necrosis by cancer cells $^{90,91}$ and is widely used in many aspects of clinical diagnosis and treatment in NSCLC. ${ }^{54,92}$ There are also many other biologic mediums available for liquid biopsy. Circulating tumor cells (CTCs) in the bloodstream were released from primary and metastatic tumor sites and have been demonstrated to be used to screen for lung cancer, detect genotyping of individual tumors, and be associated with the outcome of treatment in NSCLC. ${ }^{93,94}$ The tumorderived exosome, a new medium used for liquid biopsy, is secreted by living cells and contains nucleic acids including DNA and RNA which might reflect the tumor genomic profile and correlate with tumor dynamic in cancer patients. ${ }^{95-97}$ Except those common mediums, there are also some optimal elements including cfRNA and circulating proteins available for liquid biopsy to better guide the diagnosis and treatment selection. ${ }^{17,98}$ All of these accurate detection methods, along with the easy-to-obtain body fluid samples and those abundant mediums, will make liquid biopsy an increasingly trendy approach in the clinical management of lung cancer.

CTCs, formed by cell detachment from the primary tumor mass, and presented in the blood of many solid cancer patients, have also ever been prevalence of accurate diagnosis, prognostic, and predictive evaluation of treatment in NSCLC. Although detection of CTCs requires complex and sensitive method due to the difficulty in capturing the CTCs in circulation, ${ }^{99,100}$ the advance in technology also makes an increasing understanding of the potentials of CTCs in management of NSCLC.

Previous study provided evidence to show that CTCs detection can act as diagnostic markers in NSCLC. CTCs labeled by a folate receptor (FR) conjugate presented a sensitivity of $73.2 \%$ (67.2\% in stage I) and specificity of $84.1 \%$ to diagnose NSCLC. Moreover, CTCs also have the potential to detect genetic alterations with the accurate assay. It has been demonstrated that $92 \%$ of expected EGFR activating mutation can be detected by CTC before the course of treatment. ${ }^{101}$ Furthermore, CTCs provide another effective way to detect fusion gene identification through liquid biopsy. Tan et al ${ }^{102}$ have examined the use of FISH to analyze EML4-ALK rearrangements in comparison with matched tissue biopsies, and demonstrated $100 \%$ sensitivity. It is believed that CTCs are also capable of detecting the resistant alterations in advanced NSCLC. The agreement of detection for T790M was 74\% between CTCs and tumor biopsy. ${ }^{35}$ Extra T790M and MET amplification were identified in CTCs, but not in tumor biopsy 
from patients with PD in 1st EGFR-TKI. ${ }^{103}$ As mentioned previously, analysis of single-site tissue biopsy cannot cover the overall genomic profile related to treatment due to the inter-tumor heterogeneous. ${ }^{104}$ The same as ctDNA, CTCs can also reduce the difference in genomic profiles caused by tumor heterogeneity, as genomic analysis from a few CTCs included the overall SNV/INDEL profiles, which are shown in the different metastatic tumor sites during the course of treatment. ${ }^{105}$ Also, ctDNA combined with CTC have been proved to be complementary for assessment of acquired drug-resistance to first-line EGFRTKIs and may increase the number of patients receiving the next line precise therapy. ${ }^{103}$ As mentioned above, CTCs also provided a convenient way to evaluate the PD-L1 expression to select the proper candidates for immunotherapy. However, the rather low detection rate of CTCs limits its application for early diagnosis and variation detection. With the advance in detection methods, CTCs will be improved to a wide range of applications in clinical practice.

As a new surrogate to tumor tissue, a tumor-derived exosome has been identified that the nucleic acid in which is more stable and abundant in analysis of genetic alterations based on which seems to be more promising. It has been revealed that miRNAs isolated from exosomes enable us to distinguish between lung cancer patients and healthy individuals with a specificity and sensitivity of $92.3 \%$ and $80.3 \%$, respectively. ${ }^{106}$ The detection for common EGFR, KRAS, and BRAF mutations has higher sensitivity in plasma exoNA (exosome DNA and RNA) by NGS compared with testing of plasma ctDNA by ddPCR. ${ }^{107}$ EGFR T790M mutation was screened for both in exoNA and ctDNA of advanced NSCLC in both retrospective and prospective cohorts $(\mathrm{N}=210)$, and achieved $92 \%$ sensitivity, which is higher than in historical cohorts using ctDNA alone, and decreased the rate of unnecessary re-biopsy from $42 \%$ to $8 \%$ after getting drug resistance. ${ }^{108}$ Therefore, exosomal DNA and RNA may be used to detect mutations and explore the mechanism of resistance in NSCLC patients for its high concordance with tumor biopsy.

Circulating-free RNA has recently emerged as an important role in cancer diagnosis and response to tumor therapy. As mentioned previously, miRNA in plasma or other biologic samples can be used as biomarkers to diagnose lung cancer with accuracy. It was determined that cfRNA has the potential to predict outcomes for various therapy in NSCLC. Li et al show that increased levels of miR-660-5p in plasma after crizotinib treatment could predict a good tumor response $(P=0.012) .{ }^{109}$ Moreover, a special type of cfRNA, called tumor educated platelets (TED) related RNA and generated by confrontation between platelets and tumor cells, presented as a suitable source of non-invasive detection of cancer. A panel of RNAs was selected from platelet RNA-sequencing libraries to discriminate the early stage NSCLC and late stage NSCLC with accuracy of $81 \%$ and $88 \%$, respectively. ${ }^{98}$ The presence of cfRNA also enables the better examination of ALK rearrangements with the sensitivity of $65 \%$ sensitivity and $100 \%$ specificity by PCR based assays. ${ }^{65}$ Collectively, these evidences suggest that cfRNA may provide a robust biologic material for liquid biopsy-based diagnosis and treatment selection.

Various mediums available for liquid biopsy needs further prospective explorations and validations to determine the appropriate ones for practical situations. Particularly, the most promising biomarkers for cancer screening and the most consistent genomic profiles compared with tumors seems to be the most important factors when considered.

\section{Multiple liquid samples feasible for liquid biopsy}

Although plasma is the most popular fluid sample for liquid biopsy, there are also many other specimens available like urine, sputum, cerebrospinal fluid or pleural effusion can be obtained to address it as ctDNA and other mediums can also be found in those samples. The sensitivity of EGFR mutation in urine can reach approximately the same level as plasma test when tissue result as a reference, ${ }^{58}$ while the combination of plasma, urine, and sputum can increase the concordance of driver gene mutations in comparison with plasma only. ${ }^{110}$ The utility of the ctDNA or CTC from cerebrospinal fluid (CSF) make it possible to study the genetic alterations, and monitor treatment response as it is difficult to test the tumor site in patients with brain metastasis. CTCs are more likely to be found in CSF than in peripheral blood which provided an effective way to diagnose brain metastases in NSCLC and genetic profiles detected by CSF-CTCs were highly concordant with which in the primary tumor $(17 / 19,89.5 \%) .{ }^{111}$ Moreover, it has been illustrated that ctDNA in the CSF is more representative than plasma ctDNA and cellular CSF DNA since driver gene mutations including EGFR can be detected in all ctDNA from CSF, and the allele fractions is much higher in CSF ctDNA than in the other two media. ${ }^{79}$ Husain et $\mathrm{al}^{112}$ also indicated that driver gene mutations identified by ctDNA from ascites and pleural effusions may 
also present additional useful information not detected by tumor DNA and plasma cell-free DNA genomic profile to guide effective therapy in advanced NSCLC. This evidence was provided to reveal that unique biologic fluid samples are distinct in genomic profiles and should be applied into matched situations properly for the tumor heterogeneity. ${ }^{22}$

In spite of the appearance of considerable medium and samples for liquid biopsy, ctDNA in plasma is still the leading choice for the detection of biomarkers due to the easy access and appreciable concordance in comparison with the standard process - tumor biopsy. In order to adapt the liquid biopsy to practice efficiently, distinct liquid samples should be considered at different situations, and the differences in characters between various liquid samples needs to be clarified clearly.

\section{Conclusion}

The clinical utility of liquid sample-derived biomarkers to detect early cancer, predict tumor response and explore therapy resistance are certainly wanted and urgently required. Liquid biopsy, as an alternative way to analyze variations, not only provide a non-invasive way to detect alterations to diagnose lung cancer in advance, but also complement the result detected by tissue biopsy to allow more cancer patients to receive the precise therapy. With the increasing accurate technology, more liquid samples and mediums are available to perform comprehensive diagnosis and provide accurate treatment management for both early and advanced lung cancer.

\section{Disclosure}

The authors declare no potential conflicts of interest in this work.

\section{References}

1. Siegel RL, Miller KD, Jemal A. Cancer statistics, 2018. CA Cancer J Clin. 2018;68:7-30.

2. Torre LA, Bray F, Siegel RL, et al. Global cancer statistics. CA Cancer J Clin. 2012;65:87-108.

3. Aberle DR, Adams AM, Berg CD, et al. Reduced lung-cancer mortality with low-dose computed tomographic screening. $N$ Engl J Med. 2011;365:395-409.

4. Schwarzenbach H, Hoon DS, Pantel K. Cell-free nucleic acids as biomarkers in cancer patients. Nat Rev Cancer. 2011;11:426-437.

5. Mok TS, Wu YL, Thongprasert S, et al. Gefitinib or carboplatin-paclitaxel in pulmonary adenocarcinoma. $N$ Engl J Med. 2009;361:947-957.

6. Zhou C, Wu YL, Chen G, et al. Erlotinib versus chemotherapy as first-line treatment for patients with advanced EGFR mutation-positive non-smallcell lung cancer (OPTIMAL, CTONG-0802): a multicentre, open-label, randomised, phase 3 study. The Lancet. Oncology. 2011;12:735-742.
7. Huang $\mathrm{Q}$, Zhang $\mathrm{H}$, Hai J, et al. Impact of PD-L1 expression, driver mutations and clinical characteristics on survival after anti-PD-1/PD-L1 immunotherapy versus chemotherapy in non-small-cell lung cancer: a meta-analysis of randomized trials. Oncoimmunology. 2018;7:e1396403.

8. Goodman AM, Kato S, Bazhenova L, et al. Tumor mutational burden as an independent predictor of response to immunotherapy in diverse cancers. Mol Cancer Ther. 2017;16:2598-2608.

9. Carbone DP, Reck M, Paz-Ares L, et al. First-Line nivolumab in stage IV or recurrent non-small-cell lung cancer. $N$ Engl $J$ Med. 2017;376:2415-2426.

10. Ettinger DS, Wood DE, Aisner DL, et al. Non-small cell lung cancer, Version 5.2017, NCCN clinical practice guidelines in oncology. J Nat Compr Cancer Network. 2017;15: 504-535. doi:10.6004/jnccn.2017.0050

11. Shih JY, Gow CH, Yu CJ, et al. Epidermal growth factor receptor mutations in needle biopsy/aspiration samples predict response to gefitinib therapy and survival of patients with advanced nonsmall cell lung cancer. Int $J$ Cancer. 2006;118:963-969.

12. Chouaid C, Dujon C, Do P, et al. Feasibility and clinical impact of re-biopsy in advanced non small-cell lung cancer: a prospective multicenter study in a real-world setting (GFPC study 12-01). Lung Cancer. 2014;86:170-173.

13. Molina-Vila MA, Mayo-de-Las-Casas C, Gimenez-Capitan A, et al. Liquid biopsy in non-small cell lung cancer. Front Med. 2018;3:69.

14. Hench IB, Hench J, Tolnay M. Liquid biopsy in clinical management of breast, lung, and colorectal cancer. Front Med. 2018;5:9.

15. Leon SA, Shapiro B, Sklaroff DM, Yaros MJ. Free DNA in the serum of cancer patients and the effect of therapy. Cancer Res. 1977;37:646-650.

16. Szpechcinski A, Rudzinski P, Kupis W, et al. Plasma cell-free DNA levels and integrity in patients with chest radiological findings: NSCLC versus benign lung nodules. Cancer Lett. 2016;374:202-207.

17. Cohen JD, Li L, Wang Y, et al. Detection and localization of surgically resectable cancers with a multi-analyte blood test. Science (New York, N.Y. 2018;359:926-930.

18. Bagheri A, Khorshid HRK, Tavallaie M, et al. A panel of noncoding RNAs in non-small-cell lung cancer. $J$ Cell Biochem. 2018. doi: $10.1002 / \mathrm{jcb} .28111$

19. Lu S, Kong H, Hou Y, et al. Two plasma microRNA panels for diagnosis and subtype discrimination of lung cancer. Lung Cancer. 2018;123:44-51.

20. Gai W, Sun K. Epigenetic biomarkers in cell-free DNA and applications in liquid biopsy. Genes. 2019;10:32.

21. Weiss G, Schlegel A, Kottwitz D, Konig T, Tetzner R. Validation of the SHOX2/PTGER4 DNA methylation marker panel for plasma-based discrimination between patients with malignant and nonmalignant lung disease. J Thorac Oncol. 2018;12:77-84.

22. Shen SY, Singhania R, Fehringer G, et al. Sensitive tumour detection and classification using plasma cell-free DNA methylomes. Nature. 2018;563:579-583.

23. Yang L, Ma Y, Han W, et al. Proteinase-activated receptor 2 promotes cancer cell migration through RNA methylation-mediated repression of miR-125b. J Biol Chem. 2015;290:26627-26637.

24. Muraoka T, Soh J, Toyooka S, et al. The degree of microRNA-34b/ c methylation in serum-circulating DNA is associated with malignant pleural mesothelioma. Lung Cancer. 2013;82:485-490.

25. Ros-Mazurczyk M, Wojakowska A, Marczak L, et al. Panel of serum metabolites discriminates cancer patients and healthy participants of lung cancer screening - a pilot study. Acta Biochim Po. 2017;64:513-518

26. Fahrmann JF, Grapov D, DeFelice BC, et al. Serum phosphatidylethanolamine levels distinguish benign from malignant solitary pulmonary nodules and represent a potential diagnostic biomarker for lung cancer. Cancer Biomarkers. 2016;16:609-617. 
27. Lin $Y$, Leng Q, Jiang Z, et al. A classifier integrating plasma biomarkers and radiological characteristics for distinguishing malignant from benign pulmonary nodules. Int J Cancer. 2017;141:1240-1248.

28. Trudgen K, Khattar NH, Bensadoun E, et al. Autoantibody profiling for lung cancer screening longitudinal retrospective analysis of CT screening cohorts. PLoS One. 2014;9:e87947.

29. Ren $\mathrm{S}$, Zhang $\mathrm{S}$, Jiang $\mathrm{T}$, et al. Early detection of lung cancer by using an autoantibody panel in Chinese population. Oncoimmunology. 2007;7: e1384108.

30. Soda M, Choi YL, Enomoto M, et al. Identification of the transforming EML4-ALK fusion gene in non-small-cell lung cancer. Nature. 2007;448:561-566.

31. Horn L, Pao W. EML4-ALK: honing in on a new target in non-small-cell lung cancer. J Clin Oncol. 2009;27:4232-4235.

32. Thress KS, Paweletz CP, Felip E, et al. Acquired EGFR C797S mutation mediates resistance to AZD9291 in non-small cell lung cancer harboring EGFR T790M. Nat Med. 2015;21:560-562.

33. Coghlin CL, Smith LJ, Bakar S, et al. Quantitative analysis of tumor in bronchial biopsy specimens. $J$ Thorac Oncol. 2010;5:448-452.

34. Nosaki K, Satouchi M, Kurata T, et al. Re-biopsy status among non-small cell lung cancer patients in Japan: a retrospective study. Lung Cancer. 2016;101:1-8.

35. Sundaresan TK, Sequist LV, Heymach JV, et al. Detection of T790M, the acquired resistance EGFR mutation, by tumor biopsy versus noninvasive blood-based analyses. Clini Cancer Res. 2016;22:1103-1110.

36. Thompson JC, Yee SS, Troxel AB, et al. Detection of therapeutically targetable driver and resistance mutations in lung cancer patients by next-generation sequencing of cell-free circulating tumor DNA. Clini Cancer Res. 2016;22:5772-5782.

37. Chen S, Zhao J, Cui L, Liu Y. Urinary circulating DNA detection for dynamic tracking of EGFR mutations for NSCLC patients treated with EGFR-TKIs. Clin Transl Oncol. 2017;19:332-340.

38. Diaz LA Jr., Bardelli A. Liquid biopsies: genotyping circulating tumor DNA. J Clin Oncol. 2014;32:579-586.

39. Bai H, Mao L, Wang HS, et al. Epidermal growth factor receptor mutations in plasma DNA samples predict tumor response in Chinese patients with stages IIIB to IV non-small-cell lung cancer. J Clin Oncol. 2009;27:2653-2659.

40. Douillard JY, Ostoros G, Cobo M, et al. Gefitinib treatment in EGFR mutated caucasian NSCLC: circulating-free tumor DNA as a surrogate for determination of EGFR status. J Thorac Oncol. 2014;9:1345-1353.

41. Zhu G, Ye X, Dong Z, et al. Highly sensitive droplet digital PCR method for detection of EGFR-activating mutations in plasma cell-free DNA from patients with advanced non-small cell lung cancer. J Mol Diagn. 2015;17:265-272.

42. Wang Z, Cheng Y, An T, et al. Detection of EGFR mutations in plasma circulating tumour DNA as a selection criterion for first-line gefitinib treatment in patients with advanced lung adenocarcinoma (BENEFIT): a phase 2, single-arm, multicentre clinical trial. Lancet Respir Med. 2018;6:681-690.

43. Wang Y, Tian PW, Wang WY, et al. Noninvasive genotyping and monitoring of anaplastic lymphoma kinase (ALK) rearranged non-small cell lung cancer by capture-based next-generation sequencing. Oncotarget. 2016;7:65208-65217.

44. Cui S, Zhang W, Xiong L, et al. Use of capture-based next-generation sequencing to detect ALK fusion in plasma cell-free DNA of patients with non-small-cell lung cancer. Oncotarget. 2017;8:2771-2780.

45. Gale D, Lawson ARJ, Howarth K, et al. Development of a highly sensitive liquid biopsy platform to detect clinically-relevant cancer mutations at low allele fractions in cell-free DNA. PLoS One. 2018;13:e194630.
46. Sacher AG, Paweletz C, Dahlberg SE, et al. Prospective validation of rapid plasma genotyping for the detection of EGFR and KRAS mutations in advanced lung cancer. JAMA Oncol. 2016; 2:1014-1022.

47. Zhu YJ, Zhang HB, Liu YH, et al. Estimation of cell-free circulating EGFR mutation concentration predicts outcomes in NSCLC patients treated with EGFR-TKIs. Oncotarget. 2017;8:13195-13205.

48. Wang Y, Duan J, Chen H, et al. Analysis of EGFR mutation status in tissue and plasma for predicting response to EGFR-TKIs in advanced non-small-cell lung cancer. Oncol Lett. 2017:13:2425-2431.

49. Yang X, Zhuo M, Ye X, et al. Quantification of mutant alleles in circulating tumor DNA can predict survival in lung cancer. Oncotarget. 2016;7:20810-20824.

50. Lee JY, Qing X, Xiumin W, et al. Longitudinal monitoring of EGFR mutations in plasma predicts outcomes of NSCLC patients treated with EGFR TKIs: korean Lung Cancer Consortium (KLCC-12-02). Oncotarget. 2016;7:6984-6993.

51. Zhou Q, Yang JJ, Chen ZH, et al. Serial cfDNA assessment of response and resistance to EGFR-TKI for patients with EGFR-L858R mutant lung cancer from a prospective clinical trial. J Hematol Oncol. 2016;9:86.

52. Salazar F, Molina MA, Sanchez-Ronco M, et al. First-line therapy and methylation status of CHFR in serum influence outcome to chemotherapy versus EGFR tyrosine kinase inhibitors as second-line therapy in stage IV non-small-cell lung cancer patients. Lung Cancer. 2011;72:84-91.

53. Mau-Soerensen M, Ahlborn LB, Joenson L, et al. Dynamics of mutant BRAF V600E in free circulating DNA (fcDNA) of non-melanoma cancer patients (pts) in response to treatment with BRAF and MEK/EGFR inhibitors. J Clin Oncol. 2016;34 suppl/ 11531.

54. Zheng D, Ye X, Zhang MZ, et al. Plasma EGFR T790M ctDNA status is associated with clinical outcome in advanced NSCLC patients with acquired EGFR-TKI resistance. Sci Rep. 2016;6:20913.

55. Husain H, Kosco K, Vibat CRT, Melnikova V, Erlander MG Kinetic monitoring of EGFR T790M in urinary circulating tumor DNA to predict radiographic progression and response in patients with metastatic lung adenocarcinoma. Sante. 2015;15(2):73-80.

56. Oxnard GR, Thress KS, Alden RS, et al. Association between plasma genotyping and outcomes of treatment with osimertinib (AZD9291) in advanced non-small-cell lung cancer. J Clin Oncol. 2016;34:3375-3382.

57. Iwama E, Sakai K, Azuma K, et al. Exploration of resistance mechanisms for epidermal growth factor receptor-tyrosine kinase inhibitors based on plasma analysis by digital polymerase chain reaction and next-generation sequencing. Cancer Sci. 2018;109:3921-3933.

58. Reckamp KL, Melnikova VO, Karlovich C, et al. A highly sensitive and quantitative test platform for detection of NSCLC EGFR mutations in urine and plasma. $J$ Thorac Oncol. 2016;11: 1690-1700. doi:10.1016/j.jtho.2016.05.035

59. Oxnard GR, Hu Y, Mileham KF, et al. Assessment of resistance mechanisms and clinical implications in patients with EGFR T790M-positive lung cancer and acquired resistance to osimertinib. JAMA Oncol. 2018;4:1527-1534.

60. Thress, K.S., Markovets, A, Barrett J.C., et al. Complete clearance of plasma EFGR mutations as a predictor of outcome on osimertinib in the AURA trial. $J$ Clin Oncol. 2017;35:9018.

61. Papadimitrakopoulou VA, Collins B, Chmielecki J, et al. LBA51 Analysis of resistance mechanisms to osimertinib in patients with EGFR T790M advanced NSCLC from the AURA3 study. Ann Oncol. 2018;29 Suppl 8. 
62. Yang $\mathrm{Z}$, Yang $\mathrm{N}, \mathrm{Ou} \mathrm{Q}$, et al. Investigating novel resistance mechanisms to third-generation EGFR tyrosine kinase inhibitor osimertinib in non-small cell lung cancer patients. Clini Cancer Res. 2018;24:3097-3107.

63. Zhang Q, Zhang XC, Yang JJ, et al. EGFR L792H and G796R: two Novel Mutations Mediating Resistance to the Third-Generation EGFR Tyrosine Kinase Inhibitor Osimertinib. $J$ Thorac Oncol. 2018;13:1415-1421.

64. Ho CC, Liao WY, Lin CA, et al. Acquired BRAF V600E mutation as resistant mechanism after treatment with osimertinib. $J$ Thorac Oncol. 2017;12:567-572.

65. Nilsson RJ, Karachaliou N, Berenguer $\mathrm{J}$, et al. Rearranged EML4-ALK fusion transcripts sequester in circulating blood platelets and enable blood-based crizotinib response monitoring in non-small-cell lung cancer. Oncotarget. 2016;7:1066-1075.

66. Bordi P, Tiseo M, Rofi E, et al. Detection of ALK and KRAS mutations in circulating tumor DNA of patients with advanced ALK-Positive NSCLC with disease progression during crizotinib treatment. Clin Lung Cancer. 2017;18:692-697.

67. Katayama R, Friboulet L, Koike S, et al. Two novel ALK mutations mediate acquired resistance to the next-generation ALK inhibitor alectinib. Clini Cancer Res. 2014;20:5686-5696.

68. Ilie M, Hofman V, Dietel M, Soria JC, Hofman P. Assessment of the PD-L1 status by immunohistochemistry: challenges and perspectives for therapeutic strategies in lung cancer patients. Virchows Arch. 2016;468:511-525.

69. Nicolazzo C, Raimondi C, Mancini M, et al. Monitoring PD-L1 positive circulating tumor cells in non-small cell lung cancer patients treated with the PD-1 inhibitor Nivolumab. Sci Rep. 2016;6:31726. doi:10.1038/srep31726

70. Ilie M, Szafer-Glusman E, Hofman V, et al. Detection of PD-L1 in circulating tumor cells and white blood cells from patients with advanced non-small-cell lung cancer. Ann Oncol. 2018;29:193-199.

71. Quandt D, Dieter Zucht H, Amann A, et al. Implementing liquid biopsies into clinical decision making for cancer immunotherapy. Oncotarget. 2017;8:48507-48520.

72. Khagi Y, Goodman AM, Daniels GA, et al. Hypermutated circulating tumor DNA: correlation with response to checkpoint inhibitor-based immunotherapy. Clini Cancer Res. 2017;23:5729-5736.

73. Gandara DR, Paul SM, Kowanetz M, et al. Blood-based tumor mutational burden as a predictor of clinical benefit in non-smallcell lung cancer patients treated with atezolizumab. Nat Med. 2018;24:1441-1448.

74. Goldberg SB, Narayan A, Kole AJ, et al. Early assessment of lung cancer immunotherapy response via circulating tumor DNA. Clini Cancer Res. 2018;24:1872-1880. doi:10.1158/1078-0432.CCR-17-1341

75. Cabel L, Riva F, Servois V, et al. Circulating tumor DNA changes for early monitoring of anti-PD1 immunotherapy: a proof-ofconcept study. Ann Oncol. 2017;28:1996-2001. doi:10.1093/ annonc/mdx212

76. Ashida A, Sakaizawa K, Uhara H, Okuyama R. Circulating tumour DNA for monitoring treatment response to Anti-PD-1 immunotherapy in melanoma patients. Acta Derm Venereol. 2017;97:1212-1218.

77. Malapelle U, Pisapia P, Rocco D, et al. Next generation sequencing techniques in liquid biopsy: focus on non-small cell lung cancer patients. Transl Lung Cancer Res. 2016;5:505-510.

78. Thress KS, Brant R, Carr TH, et al. EGFR mutation detection in ctDNA from NSCLC patient plasma: A cross-platform comparison of leading technologies to support the clinical development of AZD9291. Lung Cancer. 2015;90:509-515.

79. Li YS, Jiang BY, Yang JJ, et al. Unique genetic profiles from cerebrospinal fluid cell-free DNA in leptomeningeal metastases of EGFR-mutant non-small-cell lung cancer: a new medium of liquid biopsy. Ann Oncol. 2018;29:945-952.
80. Carter J, Miller JA, Feller-Kopman D, et al. Molecular profiling of malignant pleural effusion in metastatic non-small-cell lung carcinoma. The effect of preanalytical factors. Ann Am Thorac Soc. 2015;14:1169-1176.

81. Ishii H, Azuma K, Sakai K, et al. Digital PCR analysis of plasma cell-free DNA for non-invasive detection of drug resistance mechanisms in EGFR mutant NSCLC: correlation with paired tumor samples. Oncotarget. 2015;6:30850-30858.

82. Wang W, Song Z, Zhang Y. A Comparison of ddPCR and ARMS for detecting EGFR T790M status in ctDNA from advanced NSCLC patients with acquired EGFR-TKI resistance. Cancer Med. 2017;6:154-162.

83. Cao ZY, Wu W, Hou LK, et al. [Comparison of epidermal growth factor receptor (EGFR) gene T790M mutation by droplet digital PCR and Super-ARMS PCR in plasma ctDNA samples of non-small cell lung cancer patients with the resistance to EGFR-tyrosine kinase inhibitor]. Zhonghua Bing Li Xue Za Zhi. 2018;47:910-914.

84. Blakely CM, Watkins TBK, Wu W, et al. Evolution and clinical impact of co-occurring genetic alterations in advanced-stage EGFR-mutant lung cancers. Nat Genet. 2017;49:1693-1704. doi:10.1038/ng.3990

85. Stahlberg A, Krzyzanowski PM, Egyud M, et al. Simple multiplexed PCR-based barcoding of DNA for ultrasensitive mutation detection by next-generation sequencing. Nat Protoc. 2017;12:664-682.

86. Stahlberg A, Krzyzanowski PM, Jackson JB, et al. Simple, multiplexed, PCR-based barcoding of DNA enables sensitive mutation detection in liquid biopsies using sequencing. Nucleic Acids Res. 2016;44:e105.

87. Mayo-de-Las-Casas C, Garzon Ibanez M, Jordana-Ariza N, et al. An update on liquid biopsy analysis for diagnostic and monitoring applications in non-small cell lung cancer. Expert Rev Mol Diagn. 2018;18:35-45.

88. CMS.gov. Proposed decision memo for next generation sequencing (NGS) for medicare beneficiaries with advanced cancer (CAG-00450N). Available from: https://www.cms.gov/medicarecoverage-database/details/ncaproposed-decision-memo.aspx? NCAId=290. Accessed April, 2018.

89. Mouliere F, Chandrananda D, Piskorz AM, et al. Enhanced detection of circulating tumor DNA by fragment size analysis. Sci Transl Med. 2018;10(466):eaat4921. doi: 10.1126/scitranslmed.aat 4921

90. Halicka HD, Bedner E, Darzynkiewicz Z. Segregation of RNA and separate packaging of DNA and RNA in apoptotic bodies during apoptosis. Exp Cell Res. 2000;260:248-256.

91. Li CN, Hsu HL, Wu TL, et al. Cell-free DNA is released from tumor cells upon cell death: a study of tissue cultures of tumor cell lines. J Clin Lab Anal. 2003;17:103-107.

92. An T, Cheng Y, Ding C, et al. Gefitinib as first-line treatment of plasma CtDNA EGFR mutation-positive NSCL detected by DdPCR: BENEFIT Study(CTONG 1405). WCLC, MA 11.03.

93. Calabuig-Fariñas $\mathrm{S}$, Jantus-Lewintre E, Mayo-De Las Casas C, et al. EGFR mutant cfDNA and CTC detection as biomarkers in patients diagnosed with advanced non-small cell lung cancer. $J$ Clin Oncol. 2016;34 Suppl/e23039 .

94. Zhang Z, Ramnath N, Nagrath S. Current status of CTCs as liquid biopsy in lung cancer and future directions. Front Oncol. 2015;5:209.

95. Frydrychowicz M, Kolecka-Bednarczyk A, Madejczyk M, Yasar S, Dworacki G. Exosomes - structure, biogenesis and biological role in non-small-cell lung cancer. Scand J Immunol. 2015;81:2-10.

96. Melo SA, Luecke LB, Kahlert C, et al. Glypican-1 identifies cancer exosomes and detects early pancreatic cancer. Nature. 2015;523:177-182.

97. Skog J, Wurdinger T, van Rijn S, et al. Glioblastoma microvesicles transport RNA and proteins that promote tumour growth and provide diagnostic biomarkers. Nat Cell Biol. 2008;10:1470-1476. 
98. Best MG, Sol N, In 'T Veld S, et al. Swarm intelligence-enhanced detection of non-small-cell lung cancer using tumor-educated platelets. Cancer Cell. 2017;32:238-252.e239.

99. O'Flaherty JD, Gray S, Richard D, et al. Circulating tumour cells, their role in metastasis and their clinical utility in lung cancer. Lung Cancer. 2012;76:19-25.

100. Krebs MG, Sloane R, Priest L, et al. Evaluation and prognostic significance of circulating tumor cells in patients with non-smallcell lung cancer. J Clin Oncol. 2011;29:1556-1563.

101. Maheswaran S, Sequist LV, Nagrath S, et al. Detection of mutations in EGFR in circulating lung-cancer cells. $N$ Engl J Med. 2008;359:366-377.

102. Tan CL, Lim TH, Lim TK, et al. Concordance of anaplastic lymphoma kinase (ALK) gene rearrangements between circulating tumor cells and tumor in non-small cell lung cancer. Oncotarget. 2016;7:23251-62.

103. Yanagita M, Redig AJ, Paweletz CP, et al. A prospective evaluation of circulating tumor cells and cell-free DNA in EGFR-mutant non-small cell lung cancer patients treated with Erlotinib on a phase II trial. Clini Cancer Res. 2016;22:6010-6020.

104. Meador CB, Micheel CM, Levy MA, et al. Beyond histology: translating tumor genotypes into clinically effective targeted therapies. Clini Cancer Res. 2014;20:2264-2275.

105. Ni X, Zhuo M, Su Z, et al. Reproducible copy number variation patterns among single circulating tumor cells of lung cancer patients. Proc Natl Acad Sci U S A. 2013;110:21083-21088.
106. Jin X, Chen Y, Chen H, et al. Evaluation of tumor-derived exosomal miRNA as potential diagnostic biomarkers for early-stage non-small cell lung cancer using next-generation sequencing. Clini Cancer Res. 2017;23:5311-5319.

107. Mohrmann L, Huang HJ, Hong DS, et al. Liquid biopsies using plasma exosomal nucleic acids and plasma cell-free DNA compared with clinical outcomes of patients with advanced cancers. Clini Cancer Res. 2018;24:181-188.

108. Castellanos-Rizaldos E, Grimm DG, Tadigotla V, et al. Exosomebased detection of EGFR T790M in plasma from non-small cell lung cancer patients. Clini Cancer Res. 2018;24:2944-2950.

109. Li LL, Qu LL, Fu HJ, et al. Circulating microRNAs as novel biomarkers of ALK-positive nonsmall cell lung cancer and predictors of response to crizotinib therapy. Oncotarget. 2017;8:45399-45414.

110. Wu Z, Yang W, Zhao C, Li M, Zhu L, Chen OA. 10.07 genomic profile of cell-free DNA from sputum, plasma, urine and tumor tissue and correlation with clinical effect in advanced NSCLC. $J$ Thorac Oncol. 2017;12:S1773.

111. Jiang BY, Li YS, Guo WB, et al. Detection of driver and resistance mutations in leptomeningeal metastases of NSCLC by next-generation sequencing of cerebrospinal fluid circulating tumor cells. Clini Cancer Res. 2017;23:5480-5488.

112. Husain H, Nykin D, Bui N, et al. Cell-Free DNA from ascites and pleural effusions: molecular insights into genomic aberrations and disease biology. Mol Cancer Ther. 2017;16:948-955.
OncoTargets and Therapy

\section{Publish your work in this journal}

OncoTargets and Therapy is an international, peer-reviewed, open access journal focusing on the pathological basis of all cancers, potential targets for therapy and treatment protocols employed to improve the management of cancer patients. The journal also focuses on the impact of management programs and new therapeutic agents and protocols on patient perspectives such as quality of life, adherence and satisfaction. The manuscript management system is completely online and includes a very quick and fair peer-review system, which is all easy to use. Visit http://www.dovepress.com/ testimonials.php to read real quotes from published authors. 